\title{
Chronic total occlusion without collateral blood flow does not exclude myocardial viability and subsequent recovery after revascularization
}

\author{
Jeroen J. Bax, MD, PhD, ${ }^{\mathrm{a}}$ and Victoria Delgado, $\mathrm{MD}, \mathrm{PhD}^{\mathrm{a}}$ \\ a Department of Cardiology, Heart and Lung Center, Leiden University Medical Center, Leiden, \\ The Netherlands
}

Received Mar 27, 2018; accepted Mar 27, 2018

doi: $10.1007 / \mathrm{s} 12350-018-1273-0$

\section{See related article, pp. 1720-1730}

Despite significant progress in drug development improvement, mortality in patients with ischemic heart failure remains high, specifically in medically treated patients. ${ }^{1}$ In these patients, the left ventricular ejection fraction (LVEF) is important for long-term outcome; more specifically, an LVEF $<35 \%$ is associated with high mortality.

Data from the STICH trial have shown that revascularization in these patients was associated with superior survival, ${ }^{1}$ most likely related to the improvement in LVEF noted after revascularization, or the reduction in left ventricular (LV) volumes. Alternatively, the mortality reduction may be related to revascularization of ischemic myocardium, which could prevent potentially lethal ventricular arrhythmias.

The protective effect of revascularization has been partially explained by the concept of myocardial hibernation as introduced by Professor SH Rahimtoola. ${ }^{2}$ In hibernation, chronic myocardial hypoperfusion secondary to a severe coronary artery stenosis leads to downregulation of contractile function. This phenomenon is explained by the reduced oxygen supply, with a consequent shift from aerobic to anaerobic metabolism, with less adenosine triphosphate yield, resulting in downregulation of contraction ("myocardial hibernation"). With coronary revascularization (either

Reprint requests: Jeroen J. Bax, MD, PhD, Department of Cardiology, Heart and Lung Center; Leiden University Medical Center, Albinusdreef 2, 2300 RC, Leiden; j.j.bax@lumc.nl

J Nucl Cardiol 2019;26:1731-3.

$1071-3581 / \$ 34.00$

Copyright (c) 2018 American Society of Nuclear Cardiology. with coronary artery bypass grafting or percutaneous coronary intervention), myocardial blood flow is restored with consequent reversal to aerobic metabolism and improvement of contractile function.

Different imaging techniques have been developed to detect viable myocardium, in order to predict improvement of function after revascularization. From a clinical perspective, it is therefore important to focus the viability detection on the areas of dysfunctional myocardium. One of the most frequent non-invasive imaging techniques concerns the combined assessment of resting myocardial perfusion and glucose utilization, where areas with reduced perfusion and preserved glucose utilization reflect hibernating viable myocardium. This approach has been used by Wang et $\mathrm{al}^{3}$ in the current issue of the Journal of Nuclear Cardiology, where the authors have used technetium-99m sestamibi imaging with single-photon emission computed tomography (SPECT, for resting perfusion assessment) and F18fluorodeoxyglucose (FDG, for glucose utilization) with positron emission tomography (PET). An even more complete approach integrates the assessment of hibernation (viability) with stress-inducible ischemia; with this approach stress perfusion is assessed (instead of resting perfusion) and then compared with FDG uptake. This approach does not differentiate between hibernation and stress-inducible ischemia but rather identifies the entire area of jeopardized myocardium which needs revascularization.

Various other techniques have been proposed for detection of viability, for example, dobutamine stress with echocardiography or magnetic resonance imaging (MRI), where improvement of myocardial contraction is visualized during low-dose dobutamine infusion (reflecting myocardial viability), followed by potential worsening of contraction (reflecting ischemia). An alternative is nuclear imaging with thallium-201 chloride using a stress-rest-reinjection protocol (which also 
integrates ischemia and viability assessment) or a restredistribution protocol (which reflects viability). Contrast-enhanced MRI using gadolinium identifies scar tissue with such high resolution that subendocardial scar can be discriminated from transmural scar; the non-infarcted tissue is by definition viable, but is not necessarily chemically jeopardized where revascularization is needed. Comprehensive meta-analysis of all these techniques demonstrated that the nuclear imaging techniques are the most sensitive for the detection of viable myocardium, whereas dobutamine echocardiography and MRI are the most specific. ${ }^{4}$

In the setting of a chronic total occlusion, the assessment of viability is extremely important to decide whether revascularization is needed. With chronic total occlusion scar formation occurring often, but when epicardial collateral flow is developed, the myocardium may remain viable and late revascularization may be considered. That is the topic of the article by Wang et al in the current issue of the Journal. ${ }^{3}$ The authors have collected data in 71 consecutive patients with ischemic cardiomyopathy with 122 chronic totally occluded vessels and 652 dysfunctional myocardial segments. The majority of the patients had a previous myocardial infarction (87.3\%), and did not have angina (66.2\%), but presented mainly with heart failure symptoms $(97.8 \%)$. The majority of patients had 3-vessel disease $(85.9 \%)$ with significantly reduced LVEF $(28 \pm 9 \%)$, and a severely dilated LV (end-diastolic volume $225 \pm 74 \mathrm{~mL}$, end-systolic volume $161 \pm 68 \mathrm{~mL}$ ), while $50 \%$ of patients had an LV aneurysm. In these patients, the presence/absence of collateral circulation was determined by invasive coronary angiography using the Rentrop classification ${ }^{5}$; $35(28.7 \%)$ had poor collateral circulation, $50(41.0 \%)$ had modest; and 37 $(30.3 \%)$ had good collateral circulation.

The authors have used the most sensitive technique to assess viability (hibernation) in the area of the occluded artery, and performed combined resting perfusion imaging (with technetium-99m sestamibi SPECT) and FDG PET. Moreover, scar was assessed with contrast-enhanced MRI. Interestingly, viability (hibernation) was present in around $60 \%$ in patients with angiographically poor, modest, and good collateral circulation. This is an interesting finding, and underscores that angiographic detection of collaterals is limited by spatial resolution. Similarly, non-transmural scar (as an indirect marker of viability) was also equally distributed among patients with angiographically poor, modest, and good collateral circulation (varying between $50 \%$ and $60 \%$ ). These results confirm that coronary angiography and specifically the Rentrop score are not accurate in excluding collateral flow, since to maintain viability some residual flow is needed. Conversely, it is interesting to note that the presence of collateral flow is not a guarantee for maintained viability, since approximately $40 \%$ of the areas subtended by a chronic total occlusion with collaterals did not show viability on FDG PET. This could be explained by the fact that chronic hypoperfusion may eventually result in myocyte death and scar formation. ${ }^{6}$

Subsequent revascularization was performed in 46 of $71(64.8 \%)$ patients, where recovery (defined as improvement of $\mathrm{LVEF} \geq 5 \%$ ) was noted in $27(58.7 \%)$ patients. These results confirm that areas with chronic contractile dysfunction, but maintained viability (hibernation) and/or subendocardial scar formation, may improve in function after revascularization. The sensitivity of the PET and MRI approaches reached up to $70 \%$, indicating that viability (hibernation) may not always result in functional recovery post-revascularization. This may be related to various factors. First, the mean timing of follow-up (to assess functional recovery) was 84 days (minimum 5 days, maximum 353 days); in some patients longer follow-up is needed to recover function. Second, peri-procedural damage (infarction) may have resulted in loss of viability, and graft occlusion may have prevented functional recovery. Third, when limited viability is present in patients with extensive scar formation, recovery of function may not occur.

The current results however underscore again the importance of the presence of myocardial viability (hibernation), even in patients with chronic total occlusion and absence of collateral flow on angiography; in these patients, recovery of LVEF may occur after revascularization, which may eventually translate in improved prognosis.

\section{Disclosures}

The department of Cardiology has received unrestricted research grants from Biotronik, Medtronic, Boston Scientific, and Edwards Lifesciences.

\section{References}

1. Velazquez EJ, Lee KL, Jones RH, Al-Khalidi HR, Hill JA, Panza JA, et al. Coronary artery bypass surgery in patients with ischemic cardiomyopathy. N Engl J Med. 2016;374:1511-20.

2. Rahimtoola SH. The hibernating myocardium. Am Heart J. 1989;117:211-21.

3. Wang L, Lu M, Feng L, Wang J, Fang W, He Z, et al. Relationship of myocardial hibernation, scar, and angiographic collateral flow in ischemic cardiomyopathy with coronary chronic total occlusion. J Nucl Cardiol. 2018. https://doi.org/10.1007/s12350-018-1241-8.

4. Bax JJ, Wijns W, Cornel JH, Visser FC, Boersma E, Fioretti PM. Accuracy of currently available techniques for prediction of 
functional recovery after revascularization in patients with left ventricular dysfunction due to chronic coronary artery disease; comparison of pooled data. J Am Coll Cardiol. 1997;30:1451-60.

5. Rentrop KP, Cohen M, Blanke H, Phillips RA. Changes in collateral channel filling immediately after controlled coronary artery occlusion by an angioplasty balloon in human subjects. J Am Coll Cardiol. 1985;5:587-92.

6. Schwarz ER, Schaper J, Dahl J, Altehoefer C, Grohmann B, Schoendube F, et al. Myocyte degeneration and cell death in hibernating myocardium. J Am Coll Cardiol. 1996;27:1577-85. 